\title{
An Interactive and Interpretable Interface for Diversity in Recommender Systems
}

Chun-Hua Tsai

University of Pittsburgh

cht77@pitt.edu

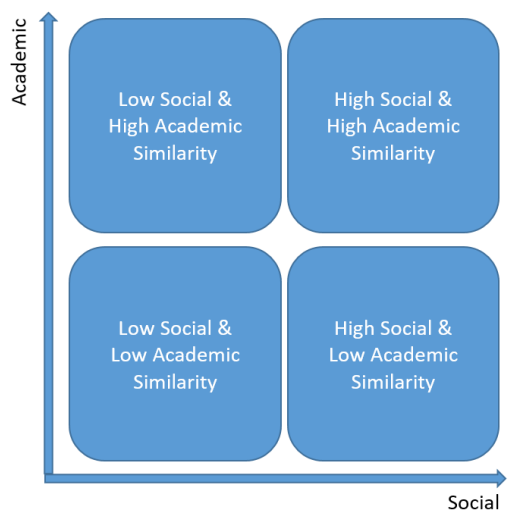

Figure 1: An example of using two dimensions (features) to show the personalized social recommendation result in four quadrants.

Permission to make digital or hard copies of part or all of this work for personal or classroom use is granted without fee provided that copies are not made or distributed for profit or commercial advantage and that copies bear this notice and the full citation on the first page. Copyrights for third-party components of this work must be honored. For all other uses, contact the owner/author(s).

Copyright held by the owner/author(s).

IUI'17 Companion, March 13-16, 2017, Limassol, Cyprus

ACM 978-1-4503-4893-5/17/03.

http://dx.doi.org/10.1145/3030024.3038292

\begin{abstract}
Offering diversity in the output of a recommender system is an active research question. Most of the current approaches focus on Top-N optimization, which results in poor user insight and accuracy trade-off. However, little is known about how an interactive interface can help with this issue. This pilot study shows that a multidimensional visualization promotes diversity among the recommended items. This finding motivated future work to provide diversity in recommender system by visualizing multivariate data through an interpretable and interactive interface.
\end{abstract}

\section{Author Keywords}

$\mathrm{HCl}$; Recommender System; Diversity

\section{ACM Classification Keywords}

H.5.m [Information interfaces and presentation (e.g., $\mathrm{HCl})]$ :

Miscellaneous

\section{ACM Copyrights \& Permission}

Accepted extended abstracts and papers will be distributed in the Conference Publications. They will also be placed in the ACM Digital Library, where they will remain accessible to thousands of researchers and practitioners worldwide. To view the ACM's copyright and permissions policy, see: http://www.acm.org/publications/policies/copyright policy. 


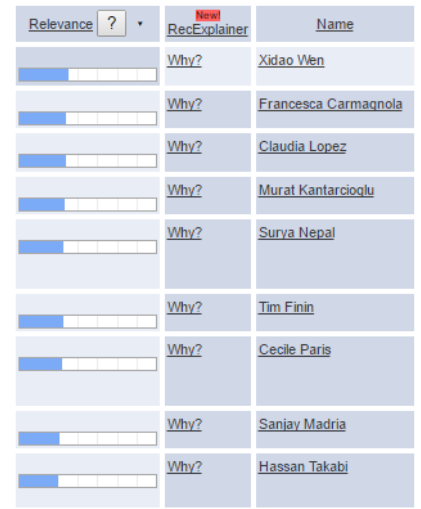

Figure 2: A Top-N list to display the personalized social

recommendation results. Each row represents an attendee at the conference. The user can click on the name to open new windows to explore the profile details.

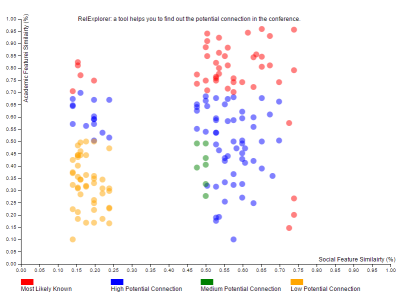

Figure 3: A two-dimensional scatterplot to display the personalized social

recommendation results. Each node represents an attendee at the conference. The user can click to open new windows to explore the profile details.

\section{Introduction}

A successful recommender system delivers useful suggestions to a user. The system usually produces a top- $\mathrm{N}$ recommendation based on collaborative or content-based

filtering approaches considering user preference or behavior. Typically, the system is concerned with the recommendation algorithm with high accuracy. However, the particular user may not always appreciate the "similar" item due to the dynamic of interests [7]. A high accuracy recommendation is exposing the user to a more narrow set of choices, which lacks diversity. The recommended results might be enhanced with more novel items or broader topic coverage to balance the diversity-accuracy dilemma [7].

An interactive interface has been proved useful to gain the user's acceptance and extend the controllability, and interpretability of recommender systems beyond the ranked list. For example, a user can adjust the slider to change the preference, profile, or source weighting for inspecting or filtering the recommended items $[5,1]$. In other words, the interface is designed to allow a user to fine-tune their preferences with real-time feedback. However, little is known about how the interactive interface can help a user to explore diversity within recommended items. A set of various choices may consist of multidimensional features, e.g. two features in Figure 1. This task is beyond an accuracy prediction regarding different user preferences or weightings. A possible solution is a user interface to display and interact with multidimensional features, e.g. an interactive scatterplot with an axis steering function [3]. The steering of axis might be useful for users to explore various items in two-dimensional features [6].

My research work is planning to answer the following research questions. 1) how to identify the explicit or implicit user desire through an interactive interface; 2 ) how to solve the challenge of displaying and exploring multidimensional features in recommender systems; and, 3) how to design and evaluate a visual recommender interface with diversity? The finding is expected to contribute to a more efficient intelligent interface of a recommender system.

\section{Progress}

This paper presents the results of a pilot study to compare the exploration patterns between the Top- $\mathrm{N}$ list and scatterplot visualization interface in a social recommender system. The system used in this pilot helps users to explore the potential connections in a conference. More specific, this system aims to make recommendations about those conference attendees with similar interests, based on both the user's preference and the relevance of other participant's work to conference attendees. The user preference was calculated using the Conference Navigator 3 system [2] and AMiner Scholarly data set ${ }^{1}$. The personalized relevance score is determined by 1) Academic Feature: the content similarity of publication text; 2) Social Feature: the distance of coauthorship network.

This system includes two interfaces to display the personalized recommendation results. One display is a Top- $\mathrm{N}$ list which ranks the conference attendees from high to low relevance based on user preference. The relevance is a linear combination of two proposed features. The user can explore the attributions through the table (Figure 2);

The second option is a scatterplot display, which shows the conference attendees as nodes in a two-dimensional space. The user can explore the nodes through two feature dimensions (Social as $\mathrm{X}$ axis and Academic as $\mathrm{Y}$ axis) (Figure 3). In the layout in Figure 1, the system divides the recommended items into four quadrants with two dimensions of social and academic features. The features

${ }^{1}$ https://aminer.org/ 


\section{User Study Overview}

Participants: Group 1: 12 participants from HT\&UMAP 2016 conference (Top-N); Group 2: 14 participants from CIC 2016 conference (Scatterplot). The participants are registered conference attendees and consisted of 10 females and 16 males. Half of them were in the 20-29 age group, while the other half of the subjects were 30-39 years age.

Task: 1) Find two conference attendees you already knew: a) decide whether you need to follow them or connect to them in the system; b) examine information about these attendees to find out how these two people can help to establish new connections at this conference; 2) Explore two conference attendees you a) don't know in person yet and b) you are interested in meeting: examine information about these attendees to find out who could introduce you to them or how you could introduce yourself to attract their interest. are normalized into cumulative probability through z-score transformations with $\mathrm{N}(0,1)$. Each quadrant is split by the 0.5 ratios, e.g. the item belongs to quadrant 2 (high academic \& social similarity) if both academic and social cumulative probability are more than 0.5 .

The user study was conducted in two international conferences to measure the following metrics [4]: 1) Diversity: the metric measured as Shannon entropy. It is defined as $D_{u}=-\sum_{q=1}^{4} p_{q} \log _{4} p_{q}$, where $p_{q}$ is the probability for the number of followed items as a proportion of all the elements in given quadrant $q$. In a layout of four quadrants, the $\log \mathrm{Base}_{4}$ is used to ensure the maximum diversity is equal to $1 ; 2$ ) Coverage: the coverage metric is defined as $C_{u}=\sum_{q=1}^{4} \frac{p_{q}}{\left|u_{q}\right|}$, where $u_{q}$ represents all items in the given quadrant $q . p_{q}$ is the number of followed items in given quadrant $q$; 3) Time: the spending time (seconds) of the task; and 4) Click: the total clicks of the task. The overview of the user study is provided in the left text box.

A non-parametric Wilcoxon signed-rank test of difference among the metrics was conducted, and they rendered a $\mathrm{W}$ value which was significant when $p<0.05$. The result is reported in Tables 1 and 2 . The experiment result indicates that the entropy and coverage metric is significantly higher among groups. This result supports the concept that scatterplot interface leads the user to explore the diverse attendees across four quadrants. This finding implies the potential of showing the recommendation in a

multidimensional view to help or change the diversity of the exploration pattern. Also, the click count is the significant difference between tasks. This result indicates it requires more investigation (clicks) when the user is asked to find the new connections (not known people). Both the group and task results reveal that the user in the scatterplot group needs more clicks to fulfill the tasks because the scatter plot does not immediately show participants' names, which initially appear in the Top-N list. However, the time metric shows an unusual pattern. The users of the scatterplot interface, which is less informative than the Top-N list, spent less time to fulfill the tasks (not statistically significant). This conflicted finding may worth future study and analysis.

\section{Proposed Approach \& Evaluation}

The preliminary experiment results support that the scatterplot display has a positive impact on the diverse exploration in a people recommendation system. Here are three possible evaluation scheme to extend the current work. I hope the consortium can provide the useful feedback on these research directions.

1) More comprehensive understanding of users' needs through an interactive interface: the interactive interface enables the user to steer the dimension axis in the desired direction through explicit interaction [3]. To support an extensive exploration, it is a challenge to learn the user's perceptions/desires through the interaction. The explicit interaction may not reflect a user's real desire if the user lacks understanding of multiple feature dimensions. For instance, an algorithm with dozens of features and classifiers would be pretty difficult to adjust sliders to learn the user's desires. It remains a challenge to design a more usable interface to let the user interact based on their implicit desires. I plan to conduct user studies to test the proposed interface and learn how users use the system to find more diverse recommendations.

2) the challenge of the multidimensional curse: the state-of-the-art recommender system usually consists of hundreds of features to deliver the highest recommendation accuracy. It creates the problem of displaying and exploring the complex data set in an interactive recommender 
Result: Between Groups

\begin{tabular}{ll}
\hline Metric & $\begin{array}{l}\text { Ave. (Group1\&2) } \\
W(p \text {-value) }\end{array}$ \\
\hline \multirow{2}{*}{ Diversity } & $\begin{array}{l}0.14 \& 0.26 \\
\end{array}$ \\
& $80(8 \mathrm{e}-06))^{* * *}$ \\
Coverage & $0.29 \& 0.53$ \\
& $30(0.005))^{* * *}$ \\
Click & $2.58 \& 4.11$ \\
& $300(0.2)$ \\
Time & $222 \& 173$ \\
& $300(0.8)$
\end{tabular}

Table 1: The experiment results: the difference between the Group 1: Top-N and Group 2: Scatterplot.

Result: Between Tasks

\begin{tabular}{|c|c|}
\hline Metric & $\begin{array}{l}\text { Ave. (Task1\&2) } \\
\text { W (p-value) }\end{array}$ \\
\hline Diversity & $\begin{array}{l}0.20 \& 0.21 \\
300(0.8)\end{array}$ \\
\hline Click & $\begin{array}{l}2.24 \& 4.52 \\
200(0.002)\end{array}$ \\
\hline Time & $\begin{array}{l}165 \& 228 \\
300(0.7)\end{array}$ \\
\hline
\end{tabular}

Table 2: The experiment results: the difference between the Task 1: Explore known connections and Task 2: Explore not known connections. interface. The most common approach is to reduce the variables to two or three dimensions through dimensional reduction, e.g. Principal component analysis (PCA). The low-dimensional variables are easier for humans to interpret. However, it creates two issues: dimension interpretability and interactivity. It requires work on how to understand the product of the dimensional reduction approaches. Also, take the human-in-a-loop into account, let the user select, label or make a decision based on the multidimensional data through the interactions. I plan to conduct a user study to test if the proposed interface is useful in helping the users to complete each task, customized to their deisres and preferences.

3) a more diverse social recommender system and its evaluation: I plan to build a social recommender system on top of the CN3 system [2]. This system helps attendees to find better connections that fit their research interests or social preferences. People exploration is a complex decision process. The user may want to explore the candidate for different reasons. For example, in a task to find a future collaborative opportunity, the user may consider more research similarity in either high (enhance) or low (complementary) publication similarity, near (local corporation) or far (cross country cooperation) geographic distance, etc. The same task but with various considerations, make the current Top- $\mathrm{N}$ list hard to fit the use cases. The future works will try to propose the system to fulfill the needs. This system will take the finding of the pilot study into account, to combine the multiple interfaces and visualizations to balance the diversity and accuracy. I will conduct the experiment at several different academic conferences, to examine if the system can deliver the value to the user for a particular desire or task.

\section{REFERENCES}

1. Svetlin Bostandjiev, John O'Donovan, and Tobias Höllerer. 2012. TasteWeights: a visual interactive hybrid recommender system. In Proceedings of the sixth ACM conference on Recommender systems. ACM, 35-42.

2. Peter Brusilovsky, Jung Sun Oh, Claudia López, Denis Parra, and Wei Jeng. 2016. Linking information and people in a social system for academic conferences. New Review of Hypermedia and Multimedia (2016), $1-31$.

3. Hannah Kim, Jaegul Choo, Haesun Park, and Alex Endert. 2016. InterAxis: Steering Scatterplot Axes via Observation-Level Interaction. IEEE transactions on visualization and computer graphics 22, 1 (2016), 131-140.

4. Jennifer Moody and David H Glass. 2016. A Novel Classification Framework for Evaluating Individual and Aggregate Diversity in Top-N Recommendations. ACM Transactions on Intelligent Systems and Technology (TIST) 7, 3 (2016), 42

5. Denis Parra and Peter Brusilovsky. 2015. User-controllable personalization: A case study with SetFusion. International Journal of Human-Computer Studies 78 (2015), 43-67.

6. David Wong, Siamak Faridani, Ephrat Bitton, Björn Hartmann, and Ken Goldberg. 2011. The diversity donut: enabling participant control over the diversity of recommended responses. In CHl'11 Extended Abstracts on Human Factors in Computing Systems. ACM, 1471-1476.

7. Cai-Nicolas Ziegler, Sean M McNee, Joseph A Konstan, and Georg Lausen. 2005. Improving recommendation lists through topic diversification. In Proceedings of the 14th international conference on World Wide Web. ACM, 22-32. 